\title{
The function of mirror neurons in the learning process
}

\author{
Daniel Mara ${ }^{1, *}$ \\ 1"Lucian Blaga" University of Sibiu, Department for Teacher Training, 34 Calea Dumbravii, Sibiu, \\ Romania
}

\begin{abstract}
In the last years, Neurosciences have developed very much, being elaborated many important theories scientific research in the field. The main goal of neuroscience is to understand how groups of neurons interact to create the behavior. Neuroscientists studying the action of molecules, genes and cells. It also explores the complex interactions involved in motion perception, thoughts, emotions and learning. Brick fundamental nervous system is the nerve cell, neuron. Neurons exchange information by sending electrical signals and chemical through connections called synapses. Discovered by a group of Italian researchers from the University of Parma, neurons - mirror are a special class of nerve cells played an important role in the direct knowledge, automatic and unconscious environment. These cortical neurons are activated not only when an action is fulfilled, but when we see how the same action is performed by someone else, they represent neural mechanism by which the actions, intentions and emotions of others can be understood automatically. In childhood neurons - mirror are extremely important. Thanks to them we learned a lot in the early years: smile, to ask for help and, in fact, all the behaviors and family and group norms. People learn by what they see and sense the others. Neurons - mirror are important to understanding the actions and intentions of other people and learn new skills through mirror image. They are involved in planning and controlling actions, abstract thinking and memory. If a child observes an action, neurons - mirror is activated and forming new neural pathways as if even he takes that action. Efficient activity of mirror neurons leads to good development in all areas at a higher emotional intelligence and the ability to empathize with others.
\end{abstract}

\section{Neurosciences}

The term of neurostiinte comes from the English neurosciences, a neoligism coined in 1962 by the American neurophysicist Francis O. Schmitt. He realised that, in order to fully understand the complexity of the brain functions, one must eliminate any existant obstacle among disciplines, by means of merging available resources and attempts.

Therefore, he used the word neurosciences (Neurosciences Research Program) to name his research team, which incorporated scientists belonging to various fields of study. The assemblage, bearing the name of neurosciences, represents an interdisciplinary branch that

\footnotetext{
* Corresponding author: daniel.mara@,ulbsibiu.ro
} 
combines pieces of information from study domains such as mathematics, physics, chemistry, nanotechnology, engineering, computer science, psychology, medicine, biology and philosophy. It follows an antipodal direction of research, when it comes to brain study and technique, than the one utilised previously.

Neurosciences cover a broad variety of aspects, such as the development, the aging process and the maintenance of the nervous system or its functional and anatomical structure, observing carefully the brain itself, along with the role it plays regarding behaviour and knowledge. Neurosciences seek to explain not only the normal mechanism of the nervous system, but the breakdowns, the malfunctions that occur in development disorders, both psychiatric and neurologic, for the purpose of finding new treatments or preventive methods.

Eric Kandel, a Nobel Prize laureate, used to say: "the main purpose of neurosciences is to explain the behaviour of the brain. How is it that the brain can direct millions of individual cells, in order to trigger a certain behaviour, and how is it that these cells can be influenced by the environment? The last barrier of neurosciences, their ultimate challenge, is to understand the biological essential features of consciousness, as well as the mental devices that allow us to perceive, react, learn and remember" [1].

\section{Mirror Neurons}

Neurosciences have encountered a great development in the last recent years and many important theories have come into existance. The main purpose of neurosciences is to understand how certain neuronal groups interact in order to create behaviour. Neurologists study the action of molecules, genes and cells, as well as the complex interplay involved in movement, perception, thought, emotions and learning. The main component of the nervous system is the brain cell, the neuron. Neurons exchange pieces of information, by means of sending electrical signals and chemical substances via the connections named synapses.

The mirror-neurons were discovered at the beginning of the 90 's by the neurophysicist Giacomo Rizzolati while studying primates and they bear this specific name because they activate in order to imitate other persons' actions and gestures. Scientists consider that there is a myriad of elements yet to be discovered, besides the physical and functional extents of mirror neurons, which require further investigations.

Giacomo Rizzolatti and his researchers have implanted electrods in the premotor cortex of several monkeys. The neurologists have noticed that the mirror neurons actuated electrical signal at the very moment when the monkeys performed movement in order to feed themselves. It was only when they noticed that the same neurons were activated when the monkeys watched other peers feeding themselves, that the scientist realised the importance of this discovery. Hence the term of mirror designated.

There are mirror-neurons that activate electrical signals in our brain when we see someone being touched, when we watch other people's movements or, only as a sign of empathy (as explained by neurologist V.S. Ramachandran and shown by the tests realised by Christian Keysers). If you watch a wounded, bleeding person, you will have a certain facial expression and experience certain certain feelings related to this situation (this is not the case for psychopaths, whose brains function differently).

The magnetic resonance imaging has shown that the same neuronal structures activate when we observe the emotional state of a human being or when we experience them ourselves, too. We can perceive mirror neurons as a way people are given the ability to connect with others (but not at a conscious level) and having as an outcome the learning process itself (learning by imitating), understanding and offering help (empathy). This connection is not realised directly, but mediated by one's personal experience. Mirror neurons help us put ourselves in other people's shoes. Yet, this neuronal system does not 
stop us of from ignoring the ones suffering, or from causing sufferance. Significant changes occur rarely and slowly, but this mechanism embedded in our brain (and therefore, in our psychology) did not appear out of the blue. There is more to this neuronal system than the evolutional function, their discovery and roles- the mirror neurons can be perceived as an indicator of the fact that we become more and more connected, at an individual or group level, or at a broader dimension, focusing on organizations and societies [2].

Mirror neurons represent a special category of brain cells, and play a very important role in the direct, automatic and instinctive understanding of the environment. These cortical neurons are activated when we observe how a specific action is performed, not only when we actually carry it out ourselves. Therefore, they become the exponents of a neuronal mechanism which helps us automatically comprehend other people's actions, intentions and feelings [3].

Mirror neurons are very important during childhood. Thanks to them, we learn a great amount of things in our early ears of life: to smile, to eat, to learn and to embrace the customs, rules and traditions of our families and groups. This is a means of learning by seeing and feeling what others see and feel. Mirror neurons are highly important when it comes to discern and assimilate other human beings' actions and intentions, and to grasp new skills by mirroring (imitating). They are involved in thought planning and controlling, in abstract thinking and memory. If a child observes an action, the mirror-neurons will activate and form new neural connections, as if he or she undertook the action herself/himself. An efficient functioning of the mirror neurons leads to an excellent development in any field, to a greater emotional intelligence and to a higher level of empathy.

It is considered that the malfunctions of these cells are linked to several ilnesses or disorders, such as autism. This fact turns the study of mirror-neurons into a very important sequence of research that could lead to finding new ways of treatment. These neurons were, initially, discovered in the premotor frontal cortex of the monkeys, and, in later studies, it was found that they were also present in the inferior parietal lobe. Their main characteristic is given by the tight connection between the motor pieces of information they codify and the visual data they respond to, bridgeing the areas of the brain in charge with both motor and sensuous processing.

According to the latest studies, the human brain also contains mirror neurons, and a large number of hypotheses were created in order to explain their functional purpose, such as understaning actions and intentions, imitating and empathizing.

The first hypothesis regarding the function of mirror neurons was the one of actions distinguishing, as peculiar as it may sound the fact that, in order to observe them, the motor system has to be activated. Actually, a simple visual perception, without the involvement of the motor system, would only offer pieces of information regarding the discernible aspects of the movement, and would not give data about its inner components or sense of direction. Approaching the issue from this point of view, mirror-neurons might be the core mechanism for a myriad of functions. Among them is the imitation, defind as the ability to reproduce or learn new actions. There are many obvius proofs that the mirror-neurons system is involved both in the directly imitation of an action performed by other people, as well as in learning behaviours by reproductions. Observing an action offers two types of pieces of information related to what and why happens, and the mirror-neurons are able not only to react to a simple stimulus, but to even understand the sense and purpose of the input, therefore being in charge with the comprehension of intentions. It was also suggested that there is a certain connection between autism and the mirror-neuronal system, the inability of autistic children to adjust to other human beings and life situations, and that this disorder might be also caused by a mirror-neurons malfunction. 
The term of Autistic Spectrum Disorder (ASD) describes a heterogenous group of neurodevelopment disorders, including the Asperger syndrome, the Rett syndrome, the Heller syndrome or the childhood disintegrative disorder, and the prervasive childhood disorder- which is not defined otherwise (typical autism). Children suffering from ASDs encounter difficulties in social interactions, verbal and non-verbal communication and show a limited range of interests and behaviours. They are often linked with repetitive, stereotypical, obsessive gestures as well as operating deficiencies. These children can also have a high level of sensitivity when it comes to sounds, smells, tastes and textures. The main three features specific to autism are: malfunctional social interactions, inability to develop relationships with peers and lack of initiative when it comes to spontaneous activities. They can also show belated speaking skills, the inability to initiate or maintain conversations, stereotypical behaviour and limited concernments, inflexible routines and repetitive motor sequences (palms and fingers movements).

ASD affect 1 in every 166 children and are four times more frequent in boys than girls. The diagnosis is usually given around the age of 2 and 3, but symptoms can be observed earlier, after 6 months. The disorder has a liniar development, without any periods of improvement, and it continues to manifest itself even at manhood, yet in less severe forms.

The ability of an individual to understand another's emotions, to perceive what the others perceives, or, in other words, to empathize, it is also closely related to mirrorneurons. The magnetic resonance imaging has shown that, while observing someone else's emotional state, the same neuronal structures are being activated as when we experience the emotions ourselves.

Some researchers connect mirror neurons with speech development, because it seems that they are located near the Broca area, region considered in charge with this function.

Representing one of the most interesting discoveries in the field of neurosciences, mirror-neurons radically change the old conceptions about human brain functioning which states that the brain works in a sequentially manner. This means that signal perception is followed by a cognitive reprocessing, a decisional one and, then, by the enforcement of the motor task.

It is quite obvious that, the ability of the specimen to react to other person's emotions and actions has a significant importance in behaviour adjustment. One could consider this statement valid because people would not efficiently work together if they did not accomodate to the emotional state of the people around them. And it is the role of the mirror-neurons to interlock our personal experiences with the ones perceived by our peers [4].

\section{The Connection Between Feuerstein's Theory and the Breakthroughs in the Field of Neurosciences}

Neurosciences can consider the biological aspects of the structural cognitive modifiability conceived by Reuven Feuerstein, by describing the cerebral mechanisms on which the process is based. The cognitive neurosciences haven't produced a set of fixed axioms, therefore, their results are seldom taken with a grain of salt. It is highly important to interpret and analyze the main results of neurosciences, which can be easily put in conjunction with other fields of study. Building on studies regarding structural cognitive modifiability, there must be endorsed a reciprocity among neurosciences and education, mainly via instrumental enrichment. There are a number of experimental and nonexperiemental researches that support instrumental enrichment, showing that, along with mediation, it promotes cognitive modificability.

Neurosciences also offer similar proof to support The Intrumental Enrichment Program. Theories provided by cognitive neurosciences about the way brain activity influences the 
brain itself, and brain activity is influenced by cerebral modification, as well as those supporting the theory of cognitive modifiability, all of them emerge from studies on brain mouldability. This is the feature of the brain that allows cognitive modifiability, and is integrated in all the stages of the learning process. The embedded mechanisms create two different functions of adjustement: the capacity to adapt to any type of environment, the ability to improve the acclimation to specific surroundings. Such characteristics of the brain are influenced by many conditional factors which are mainly of a socializing nature, and can be split into two main categories: emotions and beliefs.

The first type of modifiability, which focuses on the capacity to adapt to any type of environment, depends on the working memory and its functioning. The second one, regarding the ability to improve the acclimation to specific surroundings, relies on the formation of new models of neural systems, the core of competences. These two processes are linked together but have different roles: the working memory is required in order to create new models of neural systems, which, by their sheer nature are built via synoptic connections. Therefore, they are also created as a result of synoptic usage, requiring a cloister of the contiguous nervous tissues. Consequent to this point of view is the fact that, due to synoptic consolidation, the tasks of the working memory which involve a specific neural-map-dependent domain are being facilitated. The performance of the working memory relies on the synopic power. Even more, the entire mental activity coincides with the working memory, and the mental activities continuously blossom the brain, by means of new models of neural systems.

In spite of all these, when an organism constantly relates to the same environment, or does not learn anything new, the mental activity has the tendency to maintain the adaptive structures, avoiding the aggradizement of significant changes. When the organism confronts new situations, the mental activity immediately starts to alter the brain structures, and its ability of efficient adjustement will depend on the efficiency of synoptic intercommunication.

The working memory is an important function of such intertrades, and constitutes the cognitive component. Consequently, on one hand, the activation of the working memory has the bent for creating new models of neural systems, while, on the other hand, new models of neural systems enlarge synoptic intercommunication, creating a greater local efficienty for tge working memory.

\section{The Concept of Mediated Learning Experience}

Mediation represents the deliberate, active, intervention provided by an educator to the person he/she interracts with, aiming for a better capaciousness which also involves a gradual autonomy level.

According to the theory of modificability and mediation, at the core of the higher mental functions stays the learning process, and the role of the educator is fundamental. The forms of interaction among environment, educator and child underlie the majority of the structural changes that occur in the human cognitive apparatus. The mediator must be able to identify, choose and encompass some of the stimuli reaching the target, in order to make them more approachable. The educator will not intercede between the human being and the outside world, because his main role will be to identify the stimuli needed to be valorized in an optimal manner, to focus his/her attention on relevant data, encompassing interest, attentiveness and awareness [5].

The mediator represents a strainer for the environmental stimuli. When the learning process occurs due to alterations of the ability to adjust to the environment, mediation is supported by the capacity to co-imitate the adaptative modifications. Intentionality and reciprocity, two concepts considered by Feuerstein as the fundamental parts of mediation, 
are inherent to human relationships. The lack of mediation, or the lack of tendance, is a form of repudiating interpersonal embodiment [6].

It is important for us to emphasize the fact that, the aim of the mediated learning experience is to select, mark out and offer stimuli by manipulating the environment in which the mediated person tries to accomodate himself/herself. In other words, as long as humans adapt and then learn, not only by themselves, but also by means of social interactions, they try to connect with an exterior world. They have to explore it independently in order to embrace behaviours and mental performances which are coimitated. The cerebral activity between the mediator and the mediated person entails changes for both of the involved parts, through interplay. Each human being contiguously changes its mechanisms due to environmental interactions, by means of variation and selection, so that the brain structures configurate accordingly. Their mediation is an expression of interpersonal affiliation and, it always entails the learning process. Mediation involves inclusion and cooperation. Given this fact, mediation is always considered to be a good moment for adjustments and accomodation. Besides the individual issues of the mediator and of the mediated person, this fact also confronts the problem of environmental acclimatization.

The lack of mediation can encompass cultural poverty, yet the purport of the mediation may not pe environment-appropriate, meaning that is is not accordant to subjective learning. This is the reason why Feuerstein states that mediation does not have a specific content, because its main purpose is to enhance the behaviouristic flexibility, not to adapt only to a certain environment. This fact shows the difference between mediation and the plain cultural conduction. Throughout the process of mediation, the environment is being manipulated and harnessed according to the needs of the mediated person, and more than just the mental activities of the mediator and mediated person are being involved. The mediator also moulds the learning environment of the other human being, in order to offer as many adjustment opportunities as possible. It is quite obvious that this particular aspect grants mediation the status of a simple and educational human interaction.

The mediator percolates the environment, in order to facilitate autoplasticity, defined by Feuerstein as the way an organism changes itself, as a result of occuring environmental changes. At the other end of the spectrum is the haloplasticity, described as the set of changes meant to adjust the external reality of the organism.

Feuerstein uses the concept of mediation, or mediated learning experience, as a means of transforming the mediated person. In other words, mediation regards the manipulation of the learning environment. Its main purpose is to offer the possibility of an adaptative and educative praxis, focused on co-imitative behaviours related to the mirror-neuronal system.

\section{Conclusions}

Thanks to the discovery of the mirror-neurons a new and revolutionary research perspective has been commenced. The mirror-neurons have been, initially, detected, approximately twenty years ago and have been immediately connected to action and object recognition.

A special emphasis was put on the fact that, while observing an action conducted by another human being, the neuronal system activates as if the person itself would carry out the activity. It is considered that mirror-neurons facilitate the imitative learning process, as well as the verbal communication, and their malfunction entails disorders such as the autism [7]. 


\section{References}

1. Cosa sono le neuroscienze, Accessed 31.01. 2017

http://www.neuroscienzedipendenze.it/neuroscienze.html, (2008)

2. C. Jelea, Sistemul neuronilor oglinda, https://costinjelea.wordpress. com/2012/01/06/sistemulneuronilor-oglinda/ (2012)

3. G. Rizzolatti, C. Sinigaglia, So quel che fai. Il cervello che agisce e i neuroni specchio, (Raffaello Cortina Editore, Milano, 2008)

4. Spitalul Clinic Nicolae Malaxa, Neuronii oglinda - traducerea neurologica a empatiei si evolutiei umane,

http://www.spitalmalaxa.ro/index.php?option=com content\&view=article\&id=112:neuronii-

oglinda-traducerea-neurologica-a-empatiei-si-evolutiei-umane \&catid=45:articolemedicale\&Itemid $=222$, accessed 26 .01. 2017

5. J. Kopciowski Camerini, L'apprendimento mediato. Orientamenti teorici ed esperienze pratiche del metodo Feuerstein, (La Scuola, Brescia, 2002)

6. R. Feuerstein, J. Rand, J. Rynders, Non accettarmi come sono, (Sansoni, Milano, 1995)

7. M. Iacoboni, I neuroni specchio. Come capiamo ciò che fanno gli altri, (Bollati Boringhieri, Torino, 2008) 